\title{
Response to Afatinib in a Patient with Non-Small Cell Lung Cancer Harboring HER2 R896G Mutation: A Case Report
}

This article was published in the following Dove Press journal: OncoTargets and Therapy

\author{
Ling Lin' \\ Hongfei $\mathrm{Ge}^{2}$ \\ Zhengqing $\mathrm{Yan}^{3}$ \\ Guoqiang Wang ${ }^{3}$ \\ Xiaomai Wu (ID) \\ Dongqing $\mathrm{Lv}^{\prime}$
}

'Department of Respiratory Medicine, Taizhou Hospital of Wenzhou Medical University, Taizhou, Zhejiang 317000 , People's Republic of China; ${ }^{2}$ Department of Cardiothoracic Surgery, Taizhou Hospital of Wenzhou Medical University, Taizhou, Zhejiang 317000, People's Republic of China; ${ }^{3}$ The Medical

Department, 3D Medicines Inc., Shanghai 20II I4, People's Republic of China

Correspondence: Dongqing Lv Department of Respiratory Medicine, Taizhou Hospital of Wenzhou Medical University, 150 Ximen Street, Linhai,

Taizhou, Zhejiang 317000, People's

Republic of China

Tel +86 I38 67622009

Email Ivdq@enzemed.com
Purpose: HER 2 mutations are identified in approximately $2 \%$ of non-small-cell lung cancer (NSCLC) cases; however, until now, there are no approved standard targeted therapy for NSCLC patients harboring HER2 mutations.

Case presentation: We present a 63-year-old male with a long smoking history, who was diagnosed with stage IV squamous cell lung cancer. After the failures of two lines of treatment with carboplatin plus gemcitabine and nidaplatin plus docetaxel, in turn, the patient received a next-generation sequencing of circulating tumor DNA to seek for potential treatment opportunities. A HER2 R896G mutation was identified with an allelic fraction of $50.77 \%$. The patient received afatinib $40 \mathrm{mg}$ a day and reached a partial response after two months of treatment. The progression-free survival was more than 14 months and the treatment of afatinib was ongoing. During the treatment, treatment-related paronychia and stomatitis occurred and relieved without any management.

Conclusion: This is the first case report describing a NSCLC patient harboring a rare HER2 R896G mutation who responds to afatinib. This case suggests that afatinib might be efficacious in NSCLC patients harboring HER2 R896G mutations, and these results need to be further studied in prospective clinical trials.

Keywords: afatinib, non-small cell lung cancer, HER2 R896G, next generation sequencing

\section{Introduction}

Lung cancer remains the leading cause of cancer-related mortality worldwide. ${ }^{1}$ Nonsmall cell lung cancer (NSCLC) comprises $80 \%$ of lung cancer. Fifty percent of lung cancer patients are diagnosed with locally advanced or metastatic disease, at which point surgical resection might not be preferred. ${ }^{2}$ In recent years, with the development of genomic characterizations, several molecular-targeted therapies have brought great clinical benefits for patients harboring driver gene mutations. ${ }^{3}$ For example, tyrosine kinase inhibitors targeting epidermal growth factor receptor (EGFR) driver mutations, anaplastic lymphoma kinase $(A L K)$ rearrangement, repressor of silencing 1 (ROS1) fusions, and neurotrophic tyrosine kinase (NTRK) fusions have been approved by the Food and Drug Administration (FDA) for NSCLC patients harboring corresponding mutations. Furthermore, other oncogenic alterations, including BRAF V600E, RET, MET and HER2 have also been identified in subtypes of NSCLC and therapies targeting these mutations are underlying exploring., 4

Among these oncogenes, $H E R 2$, also known as $E r b B 2$, a sort of $E r b B$ receptor tyrosine kinase, is activated by homodimerization or heterodimerization with other 
ErbB family members, especially EGFR. ${ }^{6,7}$ The activated $H E R 2$ can subsequently activate downstream signaling molecules, including PI3K-AKT and MEK-ERK pathways. Oncogenic activation of $H E R 2$ mutations occurs in $1-4 \%$ of NSCLC, accumulating in female non-smokers with advanced lung adenocarcinoma. ${ }^{8-10}$ HER2 exon 20 in-fame insertion, especially A775_G776insYVMA, is the most common mutant form (over $80 \%$ ). ${ }^{11}$

Anti-HER2 agents have been investigated in NSCLC for a long time. In Phase II randomized study, no difference of clinical benefit was observed between gemcitabine with or without trastuzumab in HER2-positive NSCLC. ${ }^{12}$ Aado-trastuzumab emtansine (T-DM1), an antibody-drug conjugate, is composed of an anti-HER2 humanized antibody (trastuzumab) and microtubule polymerization inhibitors showed limited efficacy in HER2-positive NSCLC patients. ${ }^{13}$ However, in another Phase II study evaluating the efficacy of TDM1 in HER2-mutated NSCLC patients, the corresponding objective response rate (ORR) was $44 \%$ and the median progression-free survival (PFS) was 5 months. ${ }^{14}$ These results suggested that HER2 mutation in the protein kinase domain instead of HER2 amplification may be sensitive to anti-HER 2 agents.

Afatinib, an oral ErbB family blocker, exhibits antitumor efficacy via downregulating the phosphorylation of HER2 and EGFR. ${ }^{15}$ In previous reports, afatinib therapy has exhibited promising results and manageable profile in HER 2 exon 20 in-fame insertion, such as A775_G776insYVMA, P780_Y781ins GSP and A775_G776lnsVAG. ${ }^{16-19}$ In 2013, Mazieres and coworkers retrospectively identified 65 NSCLC harboring HER2 exon 20 insertion. Favorable results showed that the disease control rate (DCR) in patients administrated afatinib reached up to $100 \%(n=4) .{ }^{9}$ In a retrospective European EUHER2 cohort, nine patients with HER2 exon 20 insertion were treated with afatinib. The corresponding ORR and median PFS were $18.2 \%$ and 3.9 months, respectively. ${ }^{20}$ In a global NPU program, the real-world data on the use of afatinib treatment also showed the patients with HER2-mutated NSCLC harboring HER2 exon 20 A775_G776insYVMA insertion, achieved the best benefit. ${ }^{21}$ Besides of HER 2 exon 20 insertion mutations, case-reports also demonstrated the efficacy of afatinib in NSCLC patients harboring HER2 point mutations like G776L, N813D, G660R, V659E and S310Y, regardless of the mutation domain (kinase domain, transmembrane domain or extracellular domain). ${ }^{16,22-25}$ These results show promising efficacy of afatinib in NSCLC patients harboring
HER2 point mutations. However, the efficacy of afatinib in other rare HER2 mutations have been not explored.

Herein, we present a case of NSCLC patient harboring a rare HER2 R896G mutation, who responded to afatinib.

\section{Case Presentation}

A 63-year-old Chinese male with a long smoking history was diagnosed with squamous cell lung carcinoma in December 2017. The tumor node metastasis (TNM) classification of this patient was T3N2M1. The patient had multiple hepatic metastatic lesions. The Eastern Cooperative Oncology Group (ECOG) performance status score of the patient was 0 . His initial tumor biopsy was negative for EGFR mutations or ALK rearrangement according to the results from an amplification refractory mutation system-polymerase chain reaction (arms-PCR) assay. The patient was first treated with carboplatin (495 mg, day 1) and gemcitabine (1.6 g, day 1 and day 8). After the failure of the first-line treatment in May 2018 due to progressive disease (PD), the patient received nidaplatin (40 $\mathrm{mg}$ once a day, day $1-3$ ) and docetaxel $(120 \mathrm{mg}$, day 1$)$ as a second-line treatment and relapsed in July 2018.

To seek for potential treatment opportunities, circulating tumor DNA extracted from blood was subjected to DNA sequencing analysis using a cancer-gene panel with next-generation sequencing (NGS) as previously reported. ${ }^{26}$ The sequencing results showed that the patient had a HER2 exon 22 R896G mutation with an allelic fraction of $50.77 \%$ (Figure 1). Since the immunohistochemistry (IHC) detection for HER2 expression was not routinely carried out in the pathology department of our hospital, IHC examination was not performed for the patient at that time.

Thus the patient started to receive afatinib (40 mg/day) according to the NGS results in July 7, 2018. After the treatment of 2 months, the computed tomography (CT) scan showed that the maximal tumor diameter in the target lesions decreased from $5.61 \mathrm{~cm}$ to $3.34 \mathrm{~cm}$ with a decrease of $40.5 \%$ (Figure $2 \mathrm{~A}$ and $\mathrm{B}$ ). According to the response evaluation criteria in solid tumors (RESIST 1.1), this patient was evaluated as a radiological partial response (PR). Comparing the CT scan in Figure 2B, the maximal tumor diameter in (Figure $2 \mathrm{C}$ and 2D) increased from $3.31 \mathrm{~cm}$ to $3.64 \mathrm{~cm}(3.78 \mathrm{~cm})$ with an increase of $9.0 \%$ $(13.2 \%)$. Although the tumor increased, this patient was evaluated as a radiological stable disease (SD) according to the RESIST 1.1. As for the hepatic metastatic lesions, 


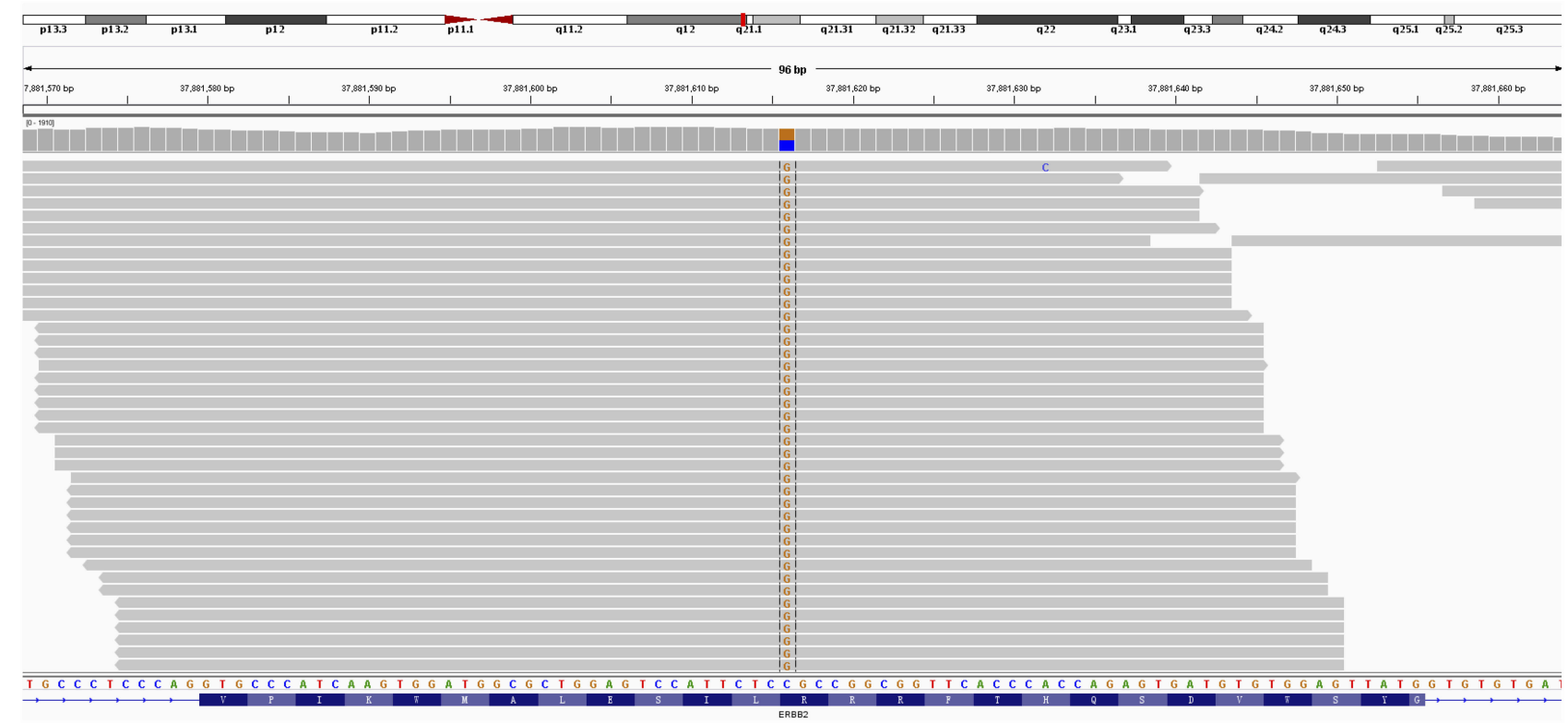

Figure I The integrative genomics viewer snapshot of HER2 R896G by next-generation sequencing.
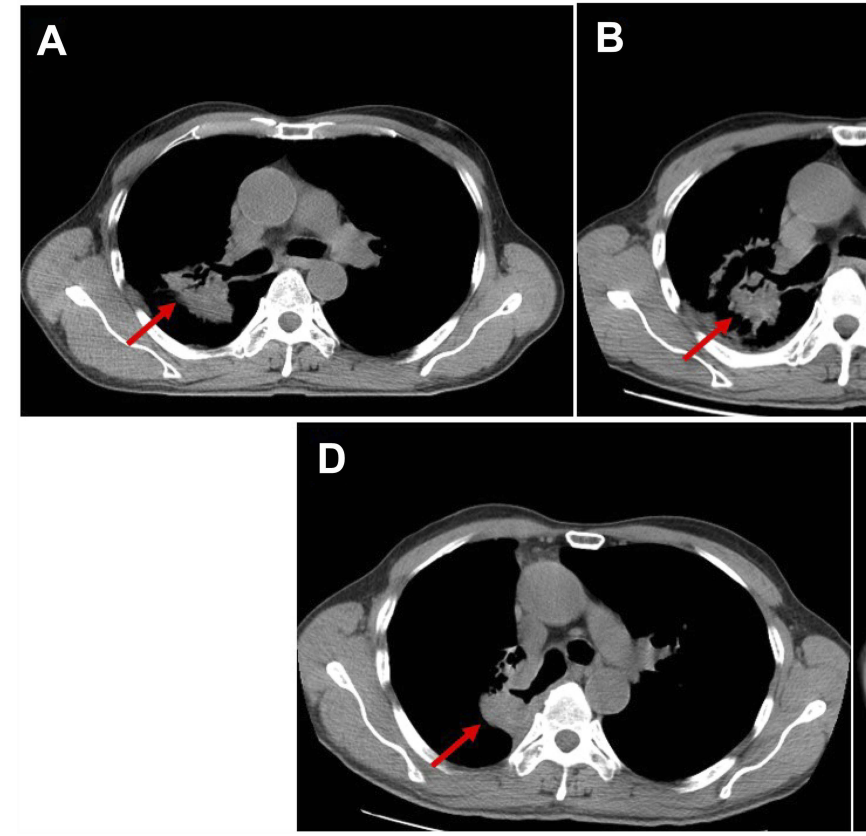
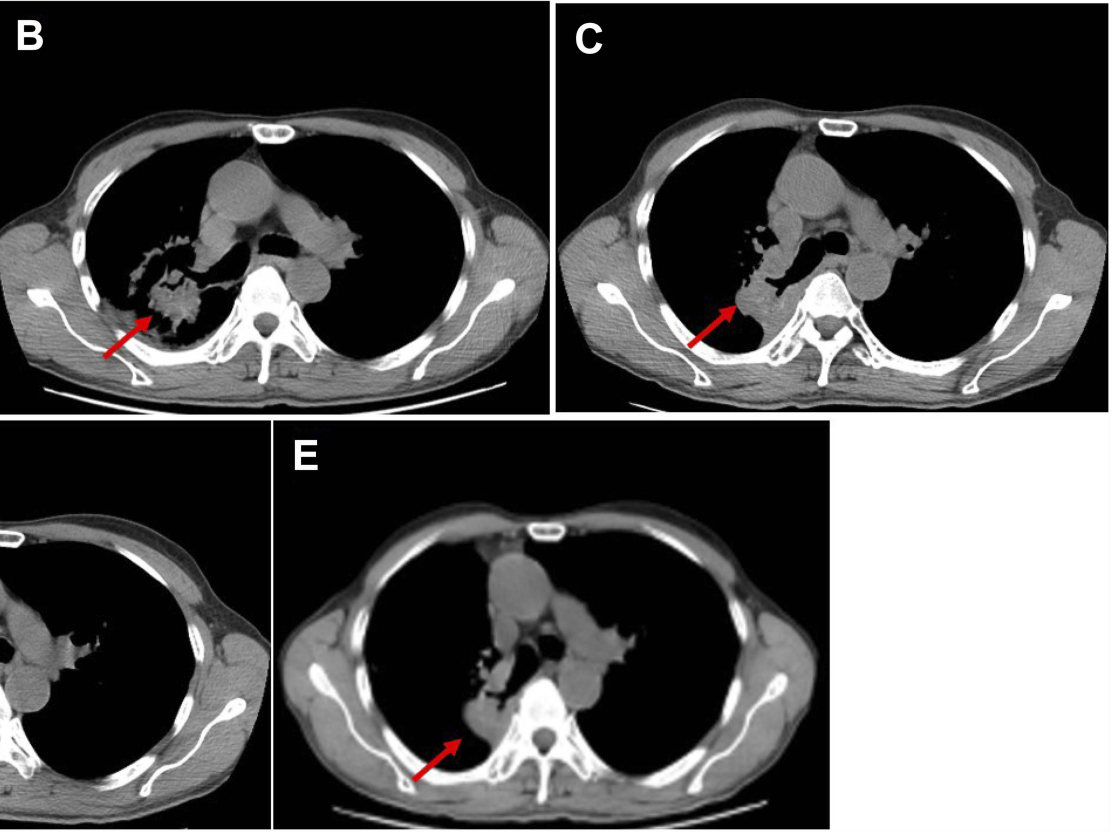

Figure 2 Computed tomography (CT) scans revealed the tumor response to afatinib. (A) The progressive disease status of lung lesion before afatinib treatment. (B) CT of the chest showing partial response after 2 month of afatinib treatment. CT of the chest showing no progressive disease after (C) 7 months, (D) II months and (E) I4 months of afatinib treatment.

the maximal tumor diameter of the biggest metastasis before afatinib treatment was $3.2 \mathrm{~cm}$ (Figure 3A). After afatinib treatment, the CT image suggested that the metastasis lesion had been reduced significantly and become unclear (Figure 3B-E). The treatment-related adverse events associated with afatinib were grade 1 paronychia and grade 1 stomatitis without treatment interruption, which occurred during the first month of afatinib treatment and relieved without any treatment. Moreover, he got fever and obstructive pulmonary disease during the treatment, but it was hard to judge whether these symptoms were related with afatinib. Until September 2019, the latest return visit, no progressive disease had been observed in the patient (Figure 2E). The progression-free survival is 

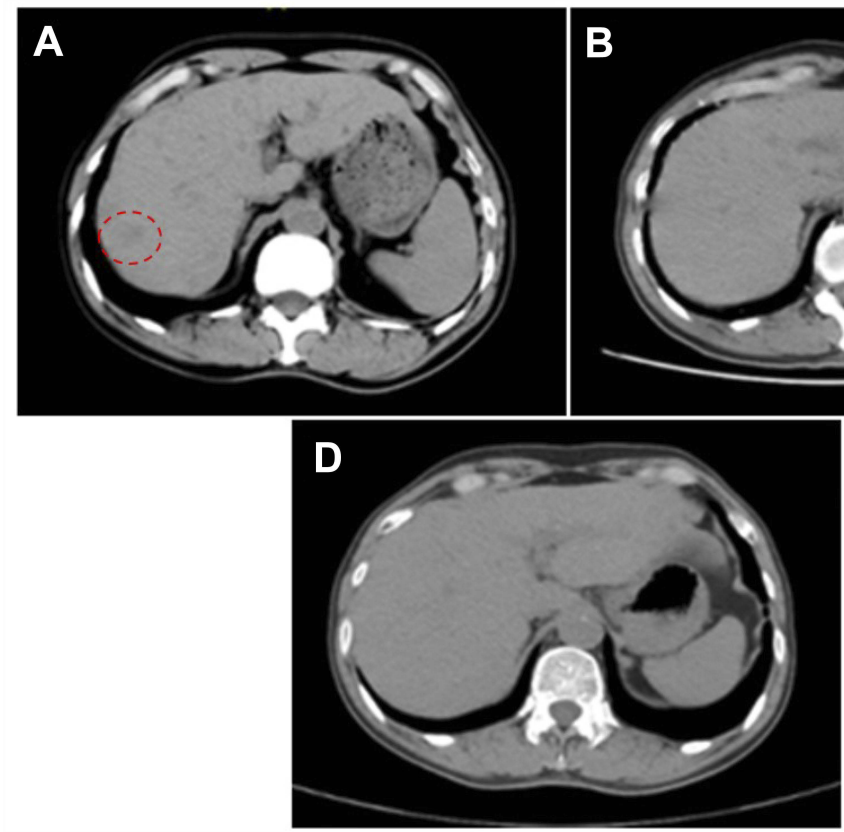
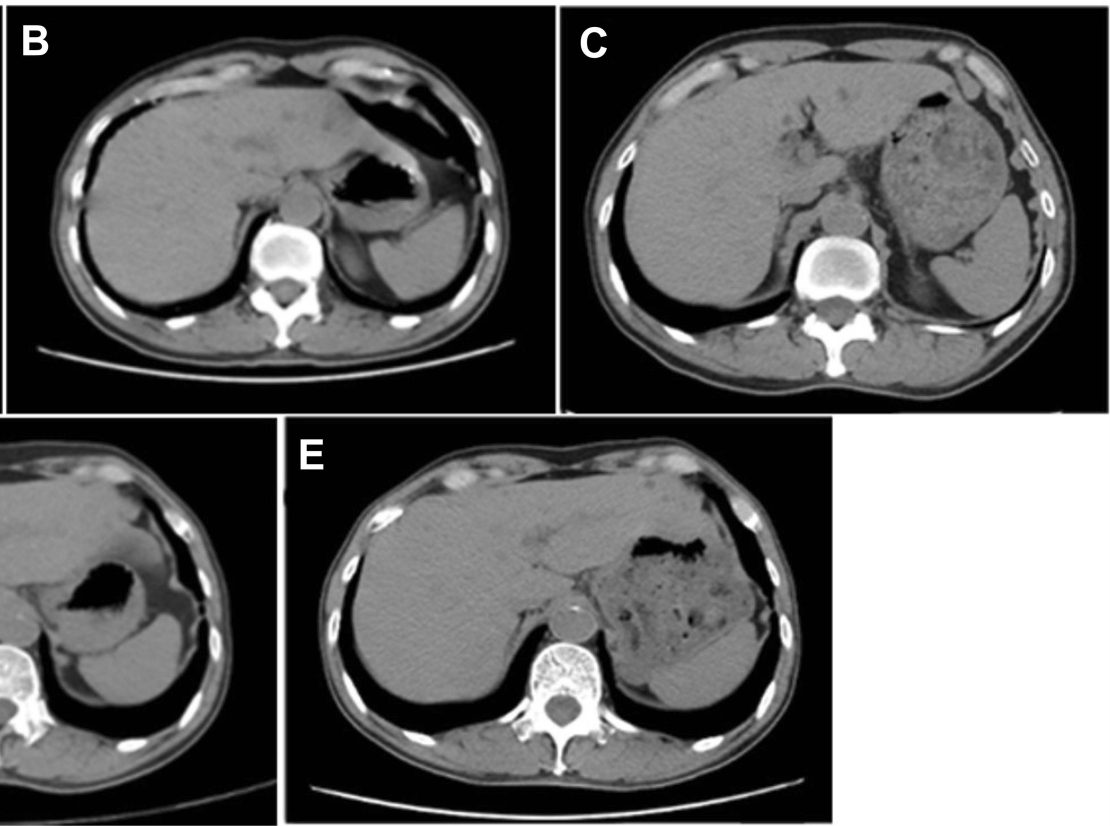

Figure 3 Computed tomography (CT) scans revealed the changes of the metastatic lesion (A) before afatinib treatment and after (B) 2 month, (C) 7 months, (D) II months and (E) 14 months of afatinib treatment. The red dash circle in (A) represents the biggest metastasis lesion.

more than 14 months. Thus far, the afatinib treatment is ongoing.

This study was approved by the Medical Ethics Committee of the Taizhou Hospital of Wenzhou Medical University. Written informed consent had also been provided by the patient for publishing the case details and accompanying images in the case study.

\section{Discussion}

In this case, we discussed a 63-year-old male diagnosed with stage IV squamous cell lung cancer. After the failures of two lines of chemotherapy, the patient was found to harbor a HER2 R896G mutation by a next-generation sequencing of circulating tumor DNA. Radiologically, the patient responded very well to afatinib, suggesting that afatinib may a potential treatment option for NSCLC patients harboring HER2 R896G mutation.

In previous reports, NSCLC patients harboring HER2 mutations are mainly female non-smokers with the adenocarcinoma subtype. ${ }^{8}$ Among these patients with HER2-mutated NSCLC, the most common HER2 mutations are exon 20 infame insertion, such as A775_G776insYVMA and P780_Y781ins GSP. ${ }^{11}$ Besides, some mutations in HER2 kinase domain are also documented, such as L755P, V773M, G776L E812K, N813D, R814H and Q828R. ${ }^{14,22,27}$ Though rarely occurring, the mutations in transmembrane domain $(\mathrm{G} 660 \mathrm{R} \text { and } \mathrm{V} 659 \mathrm{E})^{23}$ and extracellular domain $(\mathrm{S} 310 \mathrm{Y})^{24,25}$ have also been identified. To the best of our knowledge, HER 2 exon 22 R896G mutation has never been identified and reported. HER2 exon $22 \mathrm{R} 896 \mathrm{G}$ is located in the kinase region; thus, it is reasonable to question whether it is an activating mutation. HER2 R896C was ever recognized in an individual with breast carcinoma. ${ }^{28}$ Moreover, a preclinical experiment demonstrated that point mutation at codon 896 could increase the phosphorylation of phospholipase $\mathrm{C} \gamma{ }^{28}$ Based on these evidences mentioned above, afatinib was administered to treat the patient.

Afatinib, an oral ErbB family blocker, has been reported to display promising results in NSCLC with HER2 mutations, including A775_G776insYVMA, P780_Y781ins GSP, G776L and N813D. ${ }^{16,17,22}$ A few cases suggested that the patients with NSCLC harboring the mutations in transmembrane domain (G660R and V659E) $)^{23,29}$ and extracellular domain (S310Y) $)^{24,25}$ also can generate clinical benefit from afatinib therapy. In our case, a similar promising result and manageable toxicity profile were observed. These results suggested that afatinib may be effective in HER2 R896G.

To be mentioned, the patient received arms-PCR for EGFR and ALK sequencing when diagnosed. However, no driver mutations were identified. After progression, HER2 R896G was identified by a NGS of circulating tumor DNA, which benefited from the distinguishing features of NGS. NGS can not only uncover various types of known and unknown mutation, including single nucleotide 
variant (SNV), insertion, copy number variation $(\mathrm{CNV})$, etc., but also do multi-genic detection in a panel, which may be helpful to give more mutation information than arms-PCR. ${ }^{30}$ NGS for circulating tumor DNA detection is becoming a very important role in both clinical and research applications, because of its low cost, high accuracy, high throughput and rich information content and is recommended by National Comprehensive Cancer Network in NSCLC patients when tissues are not available.

\section{Conclusion}

Overall, we reported the first case of a patient with NSCLC harboring a rare HER2 R896G mutation, who responded to afatinib. Based on this finding, afatinib might be considered as an optimal treatment for NSCLC harboring HER2 R896G mutation. Further analysis of the efficacy of HER2targeted therapies in such cases will lay the foundation for developing optimized therapeutic regimen for NSCLC patients with uncommon driver gene mutations.

\section{Acknowledgment}

This work was supported by the Medical Science and Technology Project Foundation of Zhejiang Province of China (2015KYB439) and Scientific Research Fund of Taizhou Science and Technology Agency (1601KY05 and 1801KY19).

\section{Disclosure}

The authors report no conflicts of interest in this work.

\section{References}

1. Bray F, Ferlay J, Soerjomataram I, et al. Global cancer statistics 2018: GLOBOCAN estimates of incidence and mortality worldwide for 36 cancers in 185 countries. CA Cancer J Clin. 2018;68:394-424. doi:10.3322/caac.v68.6

2. Davidoff AJ, Tang M, Seal B, et al. Chemotherapy and survival benefit in elderly patients with advanced non-small-cell lung cancer. $J$ Clin Oncol. 2010;28:2191-2197. doi:10.1200/JCO.2009.25.4052

3. Herbst RS, Morgensztern D, Boshoff C. The biology and management of non-small cell lung cancer. Nature. 2018;553:446-454. doi:10.1038/ nature 25183

4. Wang R, Zhang Y, Pan Y, et al. Comprehensive investigation of oncogenic driver mutations in Chinese non-small cell lung cancer patients. Oncotarget. 2015;6:34300-34308. doi:10.18632/oncotarget. 5549

5. Li T, Kung HJ, Mack PC, et al. Genotyping and genomic profiling of non-small-cell lung cancer: implications for current and future therapies. J Clin Oncol. 2013;31:1039-1049. doi:10.1200/JCO.2012. 45.3753

6. Kosaka T, Tanizaki J, Paranal RM, et al. Response heterogeneity of EGFR and HER2 exon 20 insertions to covalent EGFR and HER2 inhibitors. Cancer Res. 2017;77:2712-2721. doi:10.1158/0008-5472. CAN-16-3404
7. Hyman DM, Piha-Paul SA, Won H, et al. HER kinase inhibition in patients with HER2- and HER3-mutant cancers. Nature. 2018;554:189-194. doi:10.1038/nature25475

8. Garrido-Castro AC, Felip E. HER2 driven non-small cell lung cancer (NSCLC): potential therapeutic approaches. Transl Lung Cancer Res. 2013;2:122-127. doi:10.3978/j.issn.2218-6751.2013.02.02

9. Mazieres J, Peters S, Lepage B, et al. Lung cancer that harbors an HER2 mutation: epidemiologic characteristics and therapeutic perspectives. J Clin Oncol. 2013;31:1997-2003. doi:10.1200/JCO.2012.45.6095

10. Song Z, Yu X, Shi Z, et al. HER2 mutations in Chinese patients with non-small cell lung cancer. Oncotarget. 2016;7:78152-78158. doi:10.18632/oncotarget.11313

11. Arcila ME, Chaft JE, Nafa K, et al. Prevalence, clinicopathologic associations, and molecular spectrum of ERBB2 (HER2) tyrosine kinase mutations in lung adenocarcinomas. Clin Cancer Res. 2012;18:4910-4918. doi:10.1158/1078-0432.CCR-12-0912

12. Gatzemeier U, Groth G, Butts C, et al. Randomized phase II trial of gemcitabine-cisplatin with or without trastuzumab in HER2-positive non-small-cell lung cancer. Ann Oncol. 2004;15:19-27. doi:10.1093/ annonc/mdh031

13. Hotta K, Aoe K, Kozuki T, et al. A Phase II study of trastuzumab emtansine in HER2-positive non-small cell lung cancer. $J$ Thorac Oncol. 2018;13:273-279. doi:10.1016/j.jtho.2017.10.032

14. Li BT, Shen R, Buonocore D, et al. Ado-trastuzumab emtansine for patients with HER2-mutant lung cancers: results from a Phase II basket trial. J Clin Oncol. 2018;36:2532-2537. doi:10.1200/JCO.2018.77.9777

15. Suzawa K, Toyooka S, Sakaguchi M, et al. Antitumor effect of afatinib, as a human epidermal growth factor receptor 2-targeted therapy, in lung cancers harboring HER2 oncogene alterations. Cancer Sci. 2016;107:45-52. doi:10.1111/cas.12845

16. De Greve J, Teugels E, Geers C, et al. Clinical activity of afatinib (BIBW 2992) in patients with lung adenocarcinoma with mutations in the kinase domain of HER2/neu. Lung Cancer. 2012;76:123-127. doi:10.1016/j.lungcan.2012.01.008

17. Shi Y, Wang M. Afatinib as first-line treatment for advanced lung adenocarcinoma patients harboring HER2 mutation: a case report and review of the literature. Thorac Cancer. 2018;9:1788-1794. doi:10.1111/tca.2018.9.issue-12

18. Costa DB, Jorge SE, Moran JP, et al. Pulse afatinib for ERBB2 exon 20 insertion-mutated lung adenocarcinomas. $J$ Thorac Oncol. 2016;11:918-923. doi:10.1016/j.jtho.2016.02.016

19. Lai WV, Lebas L, Barnes TA, et al. Afatinib in patients with metastatic or recurrent HER2-mutant lung cancers: a retrospective international multicentre study. Eur $J$ Cancer. 2019;109:28-35. doi:10.1016/j.ejca.2018.11.030

20. Mazieres J, Barlesi F, Filleron T, et al. Lung cancer patients with HER2 mutations treated with chemotherapy and HER2-targeted drugs: results from the European EUHER2 cohort. Ann Oncol. 2016;27:281-286. doi:10.1093/annonc/mdv573

21. Peters S, Curioni-Fontecedro A, Nechushtan H, et al. Activity of afatinib in heavily pretreated patients with ERBB2 mutation-positive advanced NSCLC: findings from a global named patient use program. J Thorac Oncol. 2018;13:1897-1905. doi:10.1016/j. jtho.2018.07.093

22. Park CK, Hur JY. Efficacy of afatinib in a previously-treated patient with non-small cell lung cancer harboring HER2 mutation: case report. J Korean Med Sci. 2018;33:e7. doi:10.3346/jkms.2018.33.e7

23. Herrero J, Swanton C, Ou SI, et al. HER2 Transmembrane Domain (TMD) mutations (V659/G660) that stabilize homo- and heterodimerization are rare oncogenic drivers in lung adenocarcinoma that respond to afatinib. J Thorac Oncol. 2017;12:446-457. doi:10.1016/j. jtho.2016.11.2224

24. Wang J, Wen Y, Ding G, et al. Efficacy generated by afatinib in a lung adenocarcinoma patient harboring HER2 S310Y mutation. Cancer Biol Ther. 2018;19:450-453. doi:10.1080/15384047.2018. 1449611 
25. Gao Y, Zheng A, Zhu X, et al. Clinical benefit from afatinib in an advanced squamous cell lung carcinoma patient harboring HER2 S310Y mutation: a case report. Onco Targets Ther. 2018;11:8705-8710. doi:10.2147/OTT.S182812

26. Wang Z, Duan J, Cai S, et al. Assessment of blood tumor mutational burden as a potential biomarker for immunotherapy in patients with non-small cell lung cancer with use of a next-generation sequencing cancer gene panel. JAMA Oncol. 2019;5:696. doi:10.1001/ jamaoncol.2018.7098

27. Oh IJ, Hur JY, Park CK, et al. Clinical activity of pan-HER inhibitors against HER2-mutant lung adenocarcinoma. Clin Lung Cancer. 2018;19:e775-e781. doi:10.1016/j.cllc.2018.05.018
28. Bose R, Kavuri SM, Searleman AC, et al. Activating HER2 mutations in HER2 gene amplification negative breast cancer. Cancer Discov. 2013;3:224-237. doi:10.1158/2159-8290.CD-12-0349

29. Yamamoto H, Toyooka S, Ninomiya T, et al. Therapeutic potential of afatinib for cancers with ERBB2 (HER2) transmembrane domain mutations G660D and V659E. Oncologist. 2018;23:150-154. doi:10.1634/theoncologist.2017-0345

30. Dong J, Li B, Lin D, et al. Advances in targeted therapy and immunotherapy for non-small cell lung cancer based on accurate molecular typing. Front Pharmacol. 2019;10:230.

\section{Publish your work in this journal}

OncoTargets and Therapy is an international, peer-reviewed, open access journal focusing on the pathological basis of all cancers, potential targets for therapy and treatment protocols employed to improve the management of cancer patients. The journal also focuses on the impact of management programs and new therapeutic

Submit your manuscript here: https://www.dovepress.com/oncotargets-and-therapy-journal agents and protocols on patient perspectives such as quality of life, adherence and satisfaction. The manuscript management system is completely online and includes a very quick and fair peer-review system, which is all easy to use. Visit http://www.dovepress.com/ testimonials.php to read real quotes from published authors. 